\title{
ULTRA-THIN, NON-METALLIC AND FLEXIBLE METAMATERIAL ABSORBER FOR X-BAND APPLICATIONS: DESIGN AND FABRICATION
}

\author{
Dipangkar Borah ${ }^{1}$, Nidhi S. Bhattacharyya ${ }^{2}$ \\ ${ }^{1}$ Department of Physics, Tezpur University, Tezpur - 784028, Assam, India \\ ${ }^{2}$ Department of Physics, Tezpur University, Tezpur - 784028, Assam, India
}

\begin{abstract}
With the fast advancement of wireless communications and rapid increase of number of electronic systems, electromagnetic interference (EMI) have become more prominent and hence, minimization of EMI in both civil and military applications has led to extensive research on electromagnetic absorbers. Recently, metamaterial has been studied as EMI absorbers in different frequency regime because of its unique properties that exhibits excellent result as compared to conventional absorbers in different ways. Considering the advantages of metamaterial, here we present an efficient metamaterial absorber composed of expanded graphite based conducting layers and unit cells, separated by a flexible dielectric LLDPE substrate for X-band applications. Expanded graphite is synthesized, characterized and prepared to be used as unit cells and conducting layer. Microwave characterization of linear low density polyethylene (LLDPE) is carried out in X-band and developed as the substrate for flexible metamaterial absorber. Prior to the development, a numerical simulation is carried out using CST Microwave Studio to optimize the unit cell and the proposed absorber. Experimental validation of the designed absorber is done by employing waveguide technique. The proposed absorber showing more than $90 \%$ absorption at resonance frequency $10.10 \mathrm{GHz}$. The proposed absorber possesses the advantage of being ultrathin, lightweight, flexible, corrosive resistance and can be used for different conformal applications.
\end{abstract}

Keywords: Flexible metamaterial, LLDPE substrate, expanded graphite

\section{INTRODUCTION}

In the fascinating territory of metamaterial, perfect metamaterial absorbers are the mostly invested topic among the other metamaterial based applications like, metamaterial antennas, super lenses, energy harvesting device, sensors etc [1]. For common perspective, metamaterial absorbers are basically two dimensional array of conducting resonator structures fabricated on dielectric substrate, in which the absorber characteristics purely depend only on the resonator structures [2]. Because of these designable and controllable material parameters, arising from artificial resonator structures, researchers have attracted towards the field metamaterial based absorbers in the last decades. As compared to conventional EMI absorbers, metamaterial absorber possesses advantages like thin, light weight and freedom in unit cell designing [3]. Moreover, in metamaterial absorber, by properly optimizing unit cells, composite material parameters i.e, permittivity and permeability can be deliberated to the desired value and hence their impedance matching at the air-absorber interface, which is one of the most important point while designing an EMI absorber [4].

The first perfect matematerial absorber (MMA) was presented by Landyet al. at microwave frequency in 2008, based on Veselago and Pendry's theoretical and experimental work [5-8]. His perfect absorber was capable of absorb all the incident EM waves by tuning the magnetic and electrical resonances. After the successful demonstration of Landy, different metamaterial absorbers have been developed and investigated in every frequency spectrum from radio, microwave, mm-wave, THz, MIR, NIR and optical [1]. However, in most of the reported work, metamaterials are fabricated on like, FR4, Silicon wafer substrate etc., using Copper, Gold metallic layers. These kind of substrates make the absorber rigid and unsuitable for use on non-planar surfaces like redome, body worn and health monitoring devices etc. [9]. Moreover, unit cells made up of copper and gold make the absorber corrosive and costly.

In this paper, we propose an approach of design and experimental details of a metamaterial absorber made up of flexible LLDPE substrate using expanded graphite (EG) based square unit cells. LLDPE substrate is flexible and lightweight, having low water absorption and high tensile strength. EG has almost same order of conductivity as copper and is selected for designing the square unit cells. LLDPE substrate is processed from commercially available LLDPE powders and EG is synthesized, characterized and used for design purpose. Prior to experimental fabrication, a numerical simulation is carried out to optimize the design. The fabricated sample is then tested using wave guide technique. 


\section{DESIGN AND SIMULATION}

Figure 1 shows the prospective view of proposed absorber and the unit cell with the different geometric parameters in the inset. The unit cell consists of two conducting layers separated by dielectric LLDPE substrate of dielectric constant 2.2 2.4 at $\mathrm{X}$ band. Both, top and bottom layers made up of EG conducting layer having conductivity in the order of $10^{6} \mathrm{~S} / \mathrm{m}$. The top layer is basically periodic square ring resonators laminated over LLDPE substrate, while the bottom layer is continuous conducting layer.

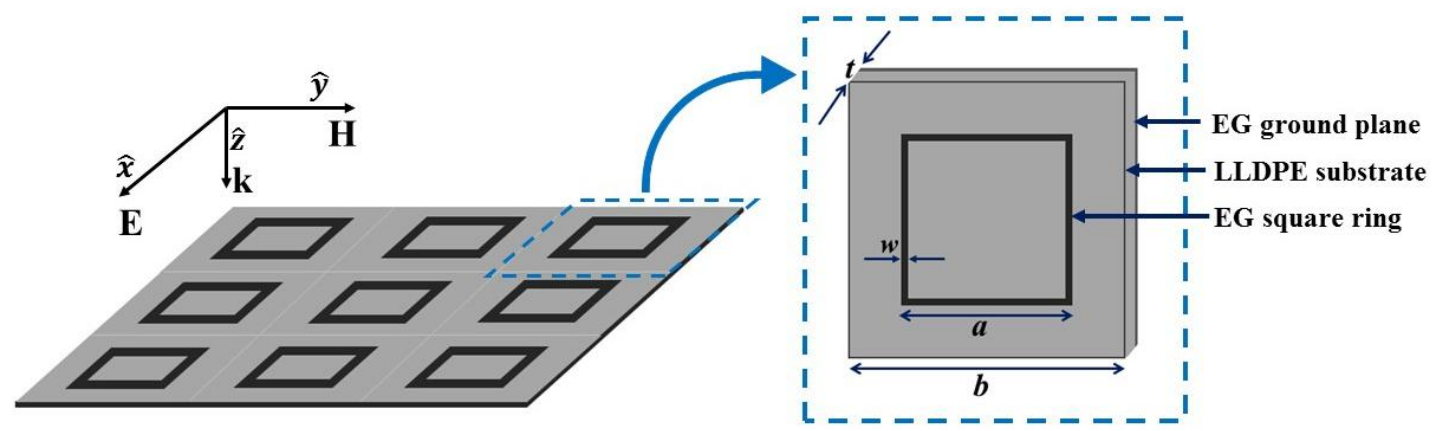

Fig.1: Schematic diagram of proposed EMI absorber

Commercially available CST Microwave studio is employed for simulation and optimization of the proposed absorber. Unit cell boundary conditions are used in frequency domain solver to simulate the proposed absorber structure. Electric, magnetic fields and direction of propagation of the incident EM wave on the absorber are shown in the Fig.1. The optimized parameters of the unit cell are presented in the Table I.

Table 1: Optimized parameters of proposed EMI absorber

\begin{tabular}{|l|l|}
\hline Unit cell parameters & Values (in mm) \\
\hline Length of square ring & 7.0 \\
\hline Width of the ring & 1.0 \\
\hline Substrate thickness & 0.5 \\
\hline Ground plane thickness & 0.1 \\
\hline Lattice constant & 12 \\
\hline
\end{tabular}

An important parameter of an absorber is its absorption, $A(\omega)$, and that can be calculated using the following formula [10],

$$
A(\omega)=1-R(\omega)-T(\omega)
$$

Where, $R(\omega)=\left|S_{11}\right|^{2}$, and $T(\omega)=\left|S_{12}\right|^{2}$ are reflectance and transmittance respectively. From equation (1), it is seen that absorption can be maximize, by minimizing the reflectance and transmittance. Reflectance is minimized by matching the impedance at air-absorber interface, while transmittance is minimized by putting conductive layer in the bottom side. The simulated reflectance and absorbance of the designed absorber is presented in the Figure 2.

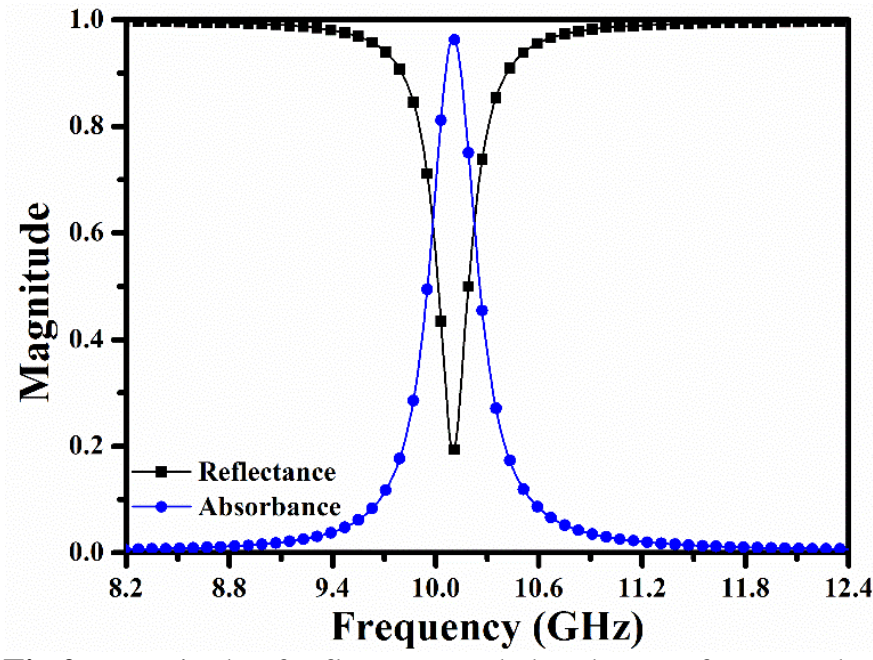

Fig.2: Magnitude of reflectance and absorbance of proposed absorber

From the Fig.2, it is observed that the proposed absorber is showing more than $90 \%$ absorption at the resonating frequency $10.10 \mathrm{GHz}$.

\section{EXPERIMENTAL DETAILS}

\subsection{Synthesis and Characterization of Expanded}

\section{Graphite}

Chemical oxidation and thermal treatment method is used to synthesize expanded graphite from natural graphite flakes [11]. For this, natural graphite flakes of size less than $2 \mu \mathrm{m}$ are purchased from Mass Graphite and Carbon products, Mehsana, India and are used to prepare expanded graphite. First, natural graphite flakes are dried at $75^{\circ} \mathrm{C}$ for 8 hours to remove the moisture contents. Dried natural graphite flakes are then mixed with saturated acid mixture that contains sulfuric and nitric acid at 3:1 volume ratio and kept for 12 hours. Sulfuric acid and nitric acid are used as intercalant and oxidizer respectively. To get uniform intercalation of 
each flake, mixture is stirred in an interval of 30 minutes. The chemically treated flakes are then washed with water, maintaining the $\mathrm{pH}$ level at seven and dried at $60^{\circ} \mathrm{C}$. Dried intercalated graphite are then exposed to high temperature in a muffle furnace maintain the temperature $800-900^{\circ} \mathrm{C}$ to form expanded graphite. Fig. 3 (a) shows the XRD pattern of the developed expanded graphite with $2 \theta$ values from $10^{\circ}$ to
$70^{0}$. The values centered at $26.62^{\circ}$ and $54.77^{\circ}$ correspond to (002) and (004) planes, which confirms the formation of expanded graphite. Scanning electron micrograph of expanded graphite is presented in the Fig.3 (b) at the magnification of $500 \mu \mathrm{m}$. It's vermicular or worm like structure indicates the opening of carbon network of natural graphite and it is basically number of graphite sheets.

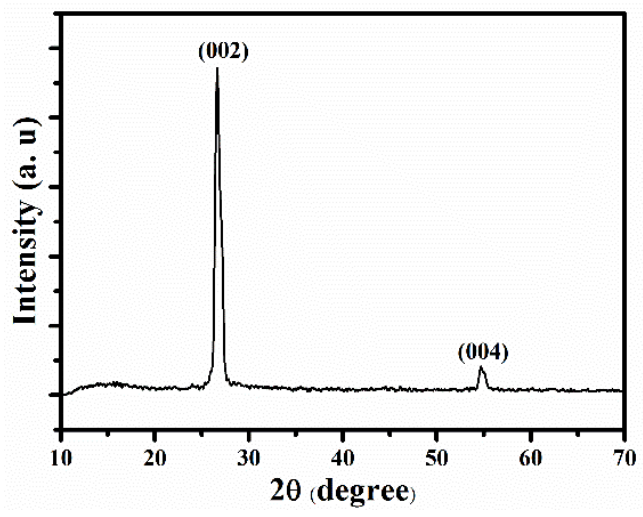

(a)

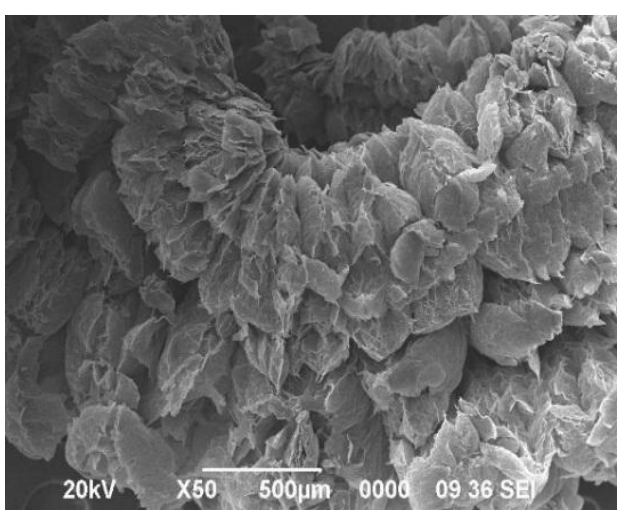

(b)

Fig.3:(a) XRD and (b) SEM image of synthesized expanded graphite

\subsection{Development of LLDPE Substrate}

Linear Low Density Polyethylene (LLDPE) powder is purchased from Sharma Plastic, India, and used without further treatment. First, LLDPE powder is kept within two steel plates and a pressure of 1 ton per $10 \mathrm{~cm}^{2}$ is applied over the steel plates with hydraulic pressure machine. The constant pressure is maintained for 10 minutes at temperature $120^{\circ} \mathrm{C}$. A spacer of thickness $0.5 \mathrm{~mm}$ is used between the steel plat to have a LLDPE substrate of thickness $0.5 \mathrm{~mm}$. Fig.4 (a) shows a LLDPE substrate, obtained after cooling down the system to room temperature and releasing the pressure gradually. Complex permittivity of the developed substrate is carried out using Agilent VNA (E8362C) at X-band frequency range and its real part is presented in the Fig.4 (b). It is seen that the real part of the complex permittivity is almost constant over the X-band.

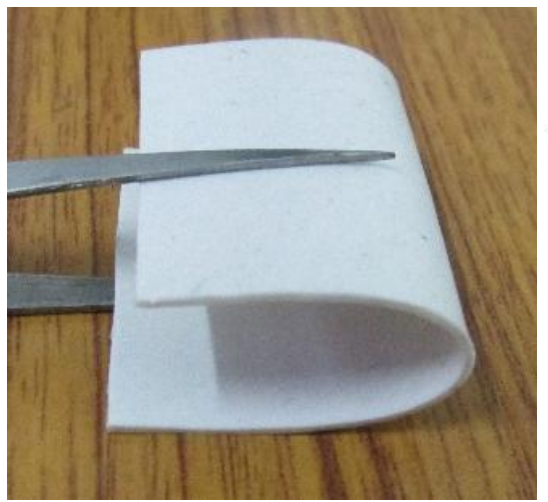

(a)

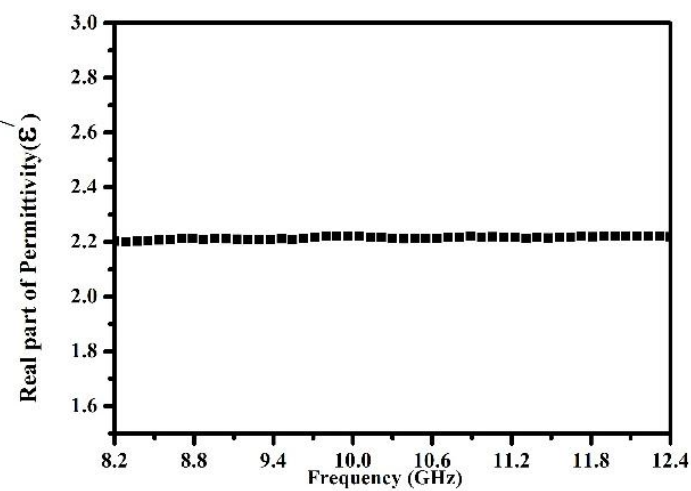

(b)

Fig.4:(a) Developed LLDPE substrate (b) real part of permittivity of LLDPE

\subsection{Fabrication of Proposed Metamaterial}

\section{Absorber and it Experimental Validation}

Synthesized EG is compressed on commercially available transparent tape to obtain a uniform EG sheet of thickness $0.1 \mathrm{~mm}$. Conductivity of the EG sheet is measured and found to be uniformed throughout the sheet. A very thin layer $\sim 0.01 \mathrm{~mm}$ of glue is spread uniformly over one side of the developed LLDPE sheet and placed over the EG sheet. The LLDPE-EG composite layer is pressed for 3 hours at room temperature for proper bonding. EG backed LLDPE substrate is shown in the Fig. 5 (a). Simple expanded graphite sheet is punctured into square ring structures and laminated over EG backed LLDPE substrate maintaining lattice parameter constant to form the proposed absorber. The developed absorber is shown in the Fig. 5 (b). 


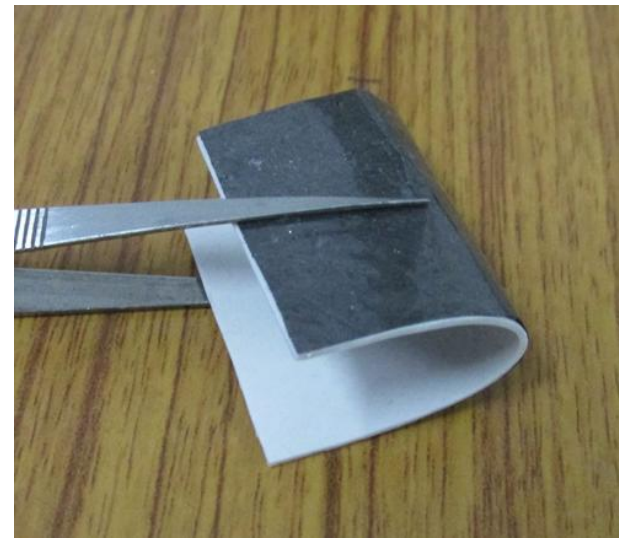

(a)

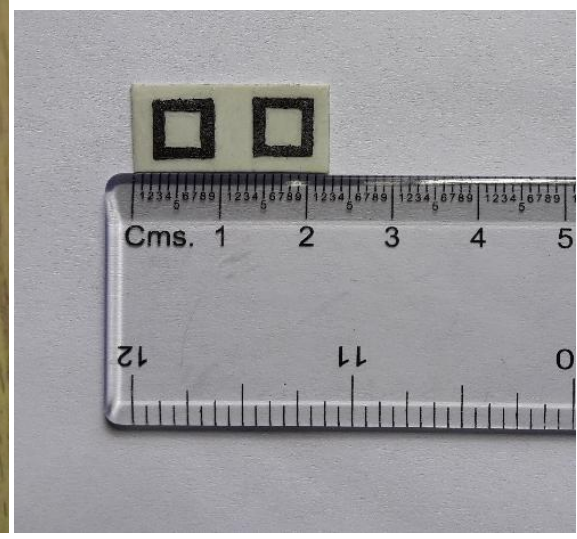

(b)

Fig.5:(a)EG backed LLDPE substrate (b) Developed absorber for waveguide measurement

Experimental characterization of the proposed absorber is carried out using waveguide method $[12,13]$. A standard Agilent X-band waveguide is connected to one port of Agilent Vector Network Analyzer (VNA) E8362C and calibrated to measure reflection loss (S11). Fabricated sample of exactly same size as the inner aperture of the standard waveguide is mounted inside the waveguide and a metal short is used to prevent any leaky wave. The fabricated sample along with the waveguide measurement systems is shown in Fig. 6 (a).The measured reflection loss is plotted the Fig. 6 (b). It is seen that experimental result in good agreement of simulated result and showing $-13.80 \mathrm{~dB}$ reflection loss at the resonating frequency $10.13 \mathrm{GHz}$. The minor discrepancy in simulated and measured result is because of edge diffraction and fabrication tolerance.

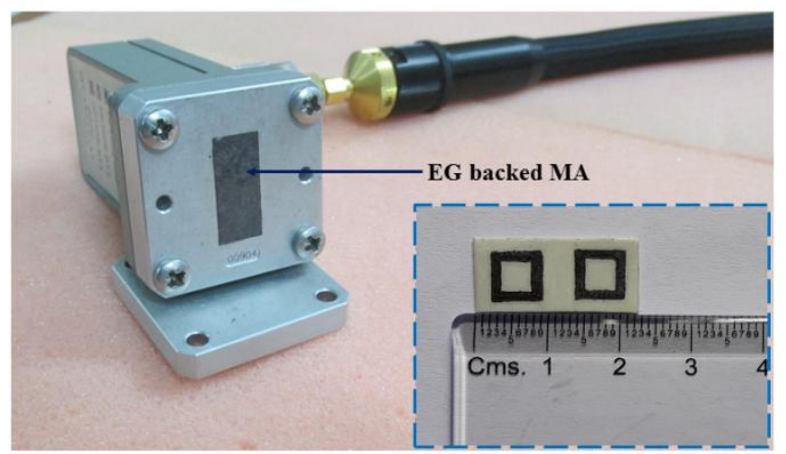

(a)

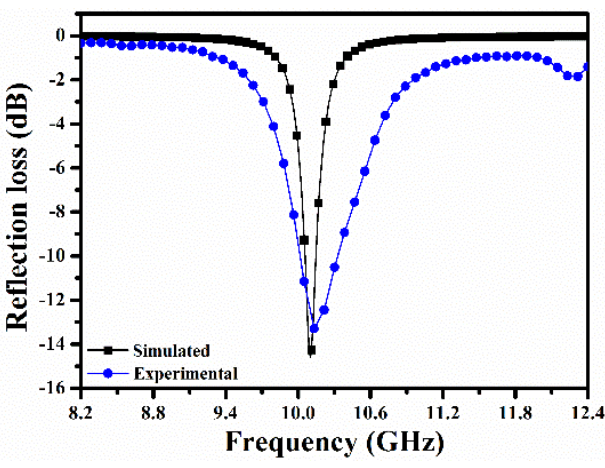

(b)

Fig.6:(a)Waveguide technique (b) measured and simulated reflection loss

The absorption nature of the proposed absorber is directlyresulted from the resonant structures of the square ring unit cells. Through simplyscaling up and down the dimensions of these structures, it is possibleto tune the absorbing frequency over a significant EM frequencyspectrum, however it is beyond the scope of this paper. Furthermore, the developed absorber has the ability to be used on curvature surfaces.

\section{DISCUSSION AND CONCLUSION}

In this paper, a metamaterial absorber for conformal application has been successfully simulated, fabricated and tested using waveguide technique. Optimized simulated absorber showing more than $90 \%$ absorption at its resonating frequency $10.10 \mathrm{GHz}$. The developed absorber shows $-13.10 \mathrm{~dB}$ reflection at $10.13 \mathrm{GHz}$ and has a thickness of $0.7 \mathrm{~mm}$. The thermal and corrosive resistance of the EG based metamaterial absorber have potential to be used in hazard condition. Considering the absorption and flexibility, thisprocessablemetamaterial absorbers may have great prospective for different EM wave absorbing applications.

\section{REFERENCES}

[1] Kollatou, T. M., Dimitriadis, A. I., Assimonis, S. D., Kantartzis, N. V., \& Antonopoulos, C. S. (2014). Multi-band, highly absorbing, microwave metamaterial structures. Applied Physics A, 115(2), 555-561.

[2] Ni, B., Chen, X. S., Huang, L. J., Ding, J. Y., Li, G. H., \& Lu, W. (2013). A dual-band polarization insensitive metamaterial absorber with split ring resonator. Optical and Quantum Electronics, 45(7), 747-753. 
[3] Li, H., Yuan, L. H., Zhou, B., Shen, X. P., Cheng, Q., \& Cui, T. J. (2011). Ultrathin multiband gigahertz metamaterial absorbers. Journal of Applied Physics, 110(1), 014909.

[4] Ozah, S., \& Bhattacharyya, N. S. (2013). Nanosized barium hexaferrite in novolac phenolic resin as microwave absorber for X-band application. Journal of Magnetism and Magnetic Materials, 342, 92-99.

[5] Landy, N. I., Sajuyigbe, S., Mock, J. J., Smith, D. R., \& Padilla, W. J. (2008). Perfect metamaterial absorber. Physical review letters, 100(20), 207402.

[6] Veselago, V. G. (1968). The electrodynamics of substances with simultaneously negative values of and?. Soviet physics uspekhi, 10(4), 509.

[7] Pendry, J. B., Holden, A. J., Stewart, W. J., \&Youngs, I. (1996). Extremely low frequency plasmons in metallic mesostructures. Physical review letters, 76(25), 4773.

[8] Pendry, J. B., Holden, A. J., Robbins, D. J., \& Stewart, W. J. (1999). Magnetism from conductors and enhanced nonlinear phenomena. IEEE transactions on microwave theory and techniques, 47(11), 2075-2084.

[9] Borah, D., \& Bhattacharyya, N. S. (2016). Design and Development of Expanded Graphite-Based Nonmetallic and Flexible Metamaterial Absorber for Xband Applications. Journal of Electronic Materials, 1-7.

[10] Bhattacharyya, S., Ghosh, S., \& Srivastava, K. V. (2013). Triple band polarization-independent metamaterial absorber with bandwidth enhancement at X-band. Journal of Applied Physics, 114(9), 094514.

[11] Gogoi, J. P., Bhattacharyya, N. S., \& Raju, K. J. (2011). Synthesis and microwave characterization of expanded graphite/novolac phenolic resin composite for microwave absorber applications. Composites Part B: Engineering, 42(5), 1291-1297.

[12] Gu, S., Barrett, J. P., Hand, T. H., Popa, B. I., \&Cummer, S. A. (2010). A broadband low-reflection metamaterial absorber. Journal of Applied Physics, 108(6), 064913.

[13] Li, L., Yang, Y., \& Liang, C. (2011). A wide-angle polarization-insensitive ultra-thin metamaterial absorber with three resonant modes. Journal of Applied Physics, 110(6), 063702. 